\title{
Stem cell-derived tissue-associated regulatory $T$ cells suppress the activity of pathogenic cells in autoimmune diabetes
}

\author{
Mohammad Haque, ${ }^{1}$ Fengyang Lei, ${ }^{2}$ Xiaofang Xiong, ${ }^{1}$ Jugal Kishore Das, ${ }^{1}$ Xingcong Ren, ${ }^{3}$ \\ Deyu Fang, ${ }^{4}$ Shahram Salek-Ardakani, ${ }^{5}$ Jin-Ming Yang, ${ }^{3}$ and Jianxun Song ${ }^{1}$ \\ 'Department of Microbial Pathogenesis and Immunology, Texas A\&M University Health Science Center, College Station, \\ Texas, USA. ${ }^{2}$ Department of Ophthalmology, Harvard Medical School, Boston, Massachusetts, USA. ${ }^{3}$ Department of \\ Pharmacology, Pennsylvania State University College of Medicine, Hershey, Pennsylvania, USA. ${ }^{4}$ Department of Pathology, \\ Northwestern University Feinberg School of Medicine, Chicago, Illinois, USA. ${ }^{5}$ Department of Pathology, Immunology and \\ Laboratory Medicine, University of Florida, Gainesville, Florida, USA.
}

\begin{abstract}
The autoantigen-specific Tregs from pluripotent stem cells (PSCs), i.e., PSC-Tregs, have the ability to suppress autoimmunity. PSC-Tregs can be programmed to be tissue associated and to infiltrate into local inflamed tissues to suppress autoimmune responses after adoptive transfer. Nevertheless, the mechanisms by which the autoantigen-specific PSC-Tregs suppress the autoimmune response remain to be fully elucidated. In this study, we generated functional autoantigen-specific Tregs from the induced PSC (iPSCs), i.e., iPSC-Tregs, and investigated the underlying mechanisms of autoimmunity suppression by these Tregs in a type 1 diabetes (T1D) murine model. A double-Tg mouse model of T1D was established in $\mathrm{F}_{1}$ mice, in which the first generation of RIP-mOVA Tg mice that were crossed with OT-I T cell receptor (TCR) Tg mice was challenged with vaccinia viruses expressing OVA (VACV-OVA). We show that adoptive transfer of OVA-specific iPSC-Tregs greatly suppressed autoimmunity in the animal model and prevented the insulin-secreting pancreatic $\beta$ cells from destruction. Further, we demonstrate that the adoptive transfer significantly reduced the expression of ICAM- 1 in the diabetic pancreas and inhibited the migration of pathogenic CD8 ${ }^{+} T$ cells and the production of the proinflammatory IFN- $\gamma$ in the pancreas. These results indicate that the stem cell-derived tissue-associated Tregs can robustly accumulate in the diabetic pancreas, and, through downregulating the expression of ICAM-1 in the local inflamed tissues and inhibiting the production of proinflammatory cytokine IFN- $\gamma$, suppress the migration and activity of the pathogenic immune cells that cause T1D.
\end{abstract}

Conflict of interest: The authors have declared that no conflict of interest exists.

Copyright: (ㄷ) 2019 American Society for Clinical Investigation

Submitted: November 27, 2018 Accepted: February 14, 2019 Published: April 4, 2019.

Reference information: /Cl Insight. 2019;4(7):e126471. https://doi. org/10.1172/jci.insight.126471.

\section{Introduction}

Type 1 diabetes (T1D) develops due to autoimmune self-destruction of pancreatic $\beta$ cells that produce insulin, and life-time administration of insulin is required for treatment of this disease (1). Although accumulating knowledge has contributed greatly to our understanding of the autoimmune pathogenesis of T1D, the precise causes remain unclear. It has been generally appreciated that autoimmune diseases arise from the breakdown of immune tolerance $(2,3)$. A number of studies found the massive infiltration of $\mathrm{CD} 8^{+} \mathrm{T}$ cells into the pancreases of newly diagnosed T1D patients whereas the number of $\mathrm{CD} 4^{+} \mathrm{T}$ cells was greatly reduced $(4,5)$. Infiltrated $\mathrm{CD} 8^{+} \mathrm{T}$ cells are pathogenic to the islets, can cause the destruction of pancreatic $\beta$ cells, and ultimately reduce the secretion of insulin.

Currently, there is no definitive treatment for controlling blood glucose level in T1D except durable insulin therapy. As a result, generation and transplantation of exogenous $\beta$ cells to replace dead or dysfunctional endogenous $\beta$ cells are considered as a promising strategy for controlling blood glucose level in patients with T1D. However, since the autoimmune disease is a continuous process, after $\beta$ cell transplantation, diabetes may develop and progress again through destruction of the pancreatic islets by pathogenic T cells. Therefore, $\beta$ cell transplantation might not be a long-lasting solution for the control of blood glucose level in T1D.

Over the past several years, there has been an increasing interest in Tregs, which play a fundamental role in controlling various autoimmune responses. Numerous preclinical studies suggest that adoptive 
transfer of Tregs can prevent or cure T cell-mediated autoimmune diseases, such as T1D and arthritis $(6,7)$. There are several advantages of the Treg adoptive transfer over conventional treatments. These advantages include (a) the potential of antigen (Ag) specificity without general immunosuppression; (b) the option of inducing "physiological" long-lasting regulation in vivo; and (c) the possibility of Treg-based immunotherapy as a customized or personally designed agent for each patient, with reduced side effects $(8,9)$. It is now generally believed that adoptive transfer of in vitro-generated Tregs can reduce the hazards of complicated surgical events throughout the life. However, the use of Tregs has been complicated due to difficulties in expanding and characterizing this minor subset of $\mathrm{T}$ cells.

Here, we report the development of a robust technique for producing a large amount of autoantigen-specific Tregs from induced pluripotent stem cells (iPSCs), i.e., iPSC-Tregs that retain all the quintessential characteristics of this $\mathrm{T}$ cell subset, including expressions of CD25, CTLA-4, and FoxP3 and production of IL-10. We show that adoptive transfer of these autoantigen-specific iPSC-Tregs significantly reduces the high ratio of $\mathrm{CD} 8^{+}$to $\mathrm{CD}^{+} \mathrm{T}$ cells in the pancreases of diabetic mice and markedly decreases the expression of intracellular adhesion molecule-1 (ICAM-1) in the pancreases of prediabetic and diabetic mice. These results demonstrate the great potential of stem cell-derived autoantigen-specific Tregs in T1D immunotherapy. Moreover, we show that the stem cell-derived tissue-associated Tregs control autoimmune diabetes via preventing the ICAM-1-mediated migration of pathogenic $\mathrm{CD}^{+} \mathrm{T}$ cells in the pancreas and suppressing the production of proinflammatory cytokine IFN- $\gamma$.

\section{Results}

Characterization of autoantigen-specific naturally occurring Treg-like iPSC-Tregs. Although FoxP3 is a master regulator and a specific molecular marker for naturally occurring Tregs (nTregs), there is evidence that FoxP3 expression is not a distinct and reliable marker or a sole regulator of functionally stable Tregs. In addition, recent evidence showed that various transcription factors are critical for the development of FoxP3 ${ }^{+}$Tregs, including Nr4a1, Ikzf4, Tnfrsf18, and Tbx21 (10). These genes, which are substantially expressed in nTregs, are essential for regulating Treg transcriptional programs and maintaining the lineage stability in Tregs.

We previously showed the generation of functional Ag-specific iPSC-Tregs, which had the ability to suppress the development of autoimmune arthritis after adoptive transfer in murine models $(11,12)$. Because the iPSC-Tregs are retrovirally transduced with FoxP3 and TCR genes and have a similar phenotype to iPSC-Tregs in expression of IL-10 and TGF- $\beta$, we examined the Treg signature genes in the autoantigen-specific iPSC-Tregs. We adoptively transferred Rag $1^{-/-}$mice with pre-iPSC-Tregs that had been cocultured with OP9-DL1/DL4/I-A ${ }^{b}$ cells for 7 days. Six weeks later, mice were sacrificed and their spleens and lymph nodes (LNs) were removed for the isolation of $\mathrm{CD} 4{ }^{+} \mathrm{CD} 25^{+}$cells. Conversely, $\mathrm{CD} 4^{+} \mathrm{CD} 25^{+}$cells were sorted from the spleens and LNs of normal C57BL/6 mice. RT-PCR analysis of the Treg signature genes showed that there were no significant differences between the mice in gene expression of the transcription factors ( Nr4a1, Il2ra, Ikzf4, Tnfrsf18, and Bclo) involved in the regulation of Treg functions (all $P>0.05$, Figure 1). Collectively, these results and suggest that the autoantigen-specific iPSC-Tregs are nTreg-like suppressive cells.

Accumulation of activated $C D 8^{+} T$ cells in autoantigen-expressing pancreases of diabetic mice. To show the expression of autoantigen in the pancreases of double-Tg mice (B6-mOVA with OT-I), we performed immunofluorescence examination. The results showed the expression of OVA autoantigen in the double-Tg mice but not in the B6 or OT-I control mice (Figure 2A). Because the OVA-specific CD8 ${ }^{+} \mathrm{T}$ cells have the ability to migrate into the pancreases of the $\mathrm{F}_{1}$ generation that results from crossing B6 mOVA with OT-I TCR Tg mice and to cause destruction of the islets, we examined the fate of OVA-specific $\mathrm{CD} 8^{+} \mathrm{T}$ cells in vivo (Figure 2B). The migrations of $\mathrm{CD} 8^{+} \mathrm{T}$ cells in diabetic mice were further confirmed by flow cytometry in which more numbers of both $\mathrm{CD}^{+}$and TCRV $\beta 5^{+}$cells were present in diabetic mice (Figure $2 \mathrm{C}$ ). In $\mathrm{F}_{1}$ mice after vaccinia virus (VACV) infection (Supplemental Figure 3; supplemental material available online with this article; https://doi.org/10.1172/jci.insight.126471DS1), the draining LNs and spleens of the $\mathrm{F}_{1}$ mice were analyzed for the presence of OT-I CD8 ${ }^{+} \mathrm{T}$ cells. The proportions of OVA-specific CD8 ${ }^{+}$cells among the total $\mathrm{CD}^{+}$cells in the pancreatic LNs were significantly higher than in the pyloric, mesenteric, inguinal, cervical, or splenic LNs. No apparent accumulation or homing was observed in the non-Tg control B6 mice (Figure 2D). Additionally, all $\mathrm{F}_{1}$ mice after VACV infection developed autoimmune diabetes at age of 9 weeks, which was confirmed by the measurement of blood sugar (Figure $2 \mathrm{E}$ ). These results verify that autoantigen-specific $\mathrm{CD}^{+} \mathrm{T}$ cells are the main pathogenic immune cells that induce autoimmune diabetes in the murine model. 
A

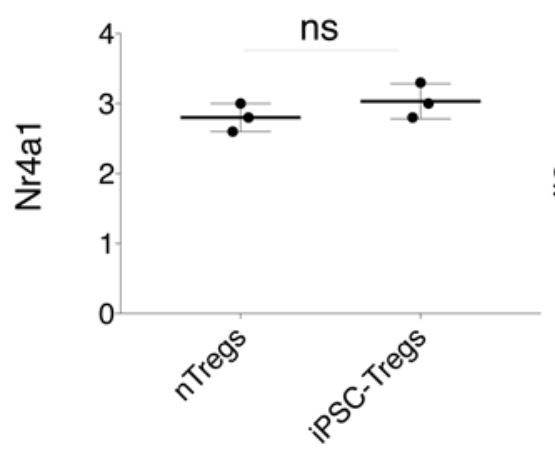

D

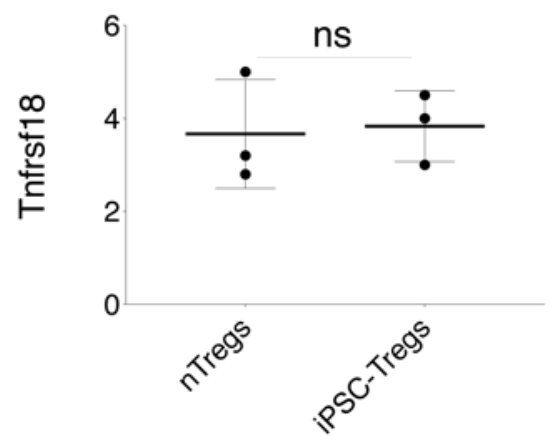

B

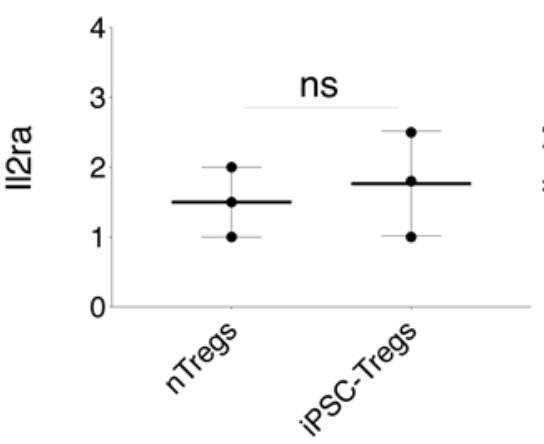

E

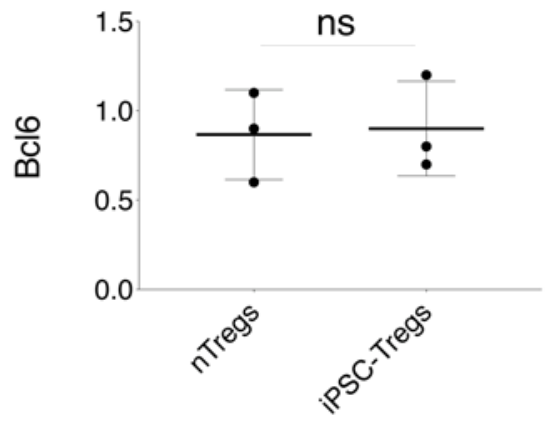

C

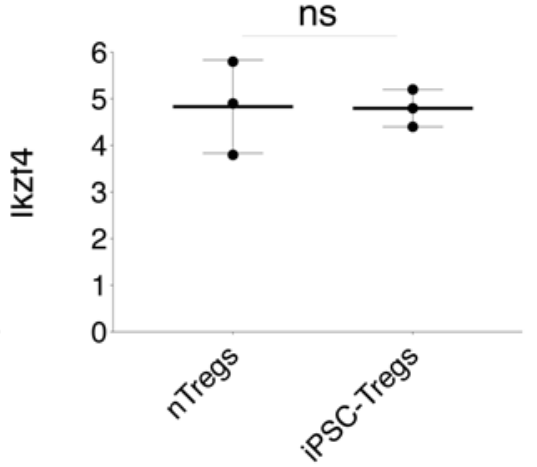

Figure 1. Characterization of autoantigen-specific $\mathbf{n T r e g - l i k e ~ i P S C - T r e g s . ~ P C R ~ a n a l y s i s ~ w a s ~ p e r f o r m e d ~ b y ~ T a q M a n ~ r e a l - t i m e ~ P C R . ~ P r i m e r s ~ f o r ~ s e q u e n c e s ~}$ were used as follows: (A) Nr4a1, forward 5'-TGTCAGGGCTGCAAGGGCTTC-3', reverse 5'-AAGCGGCAGAACTGGCAGCGG-3'; (B) II2ra, forward 5'-AACTGCCAGTGCACCAGCAAC-3', reverse 5' GAGGTCGCTCCCTCCAGTCAC-3'; (C) Ikzf4, forward 5'-CAATCTCCTTCGCCACATCAAG-3', reverse 5'-CCCACAGTAGTTCCACTTGTAG-3'; (D) Tbx21, forward 5'-GGAGCCCACTGGATGCGCCAG-3', reverse 5' AGGCAGCCTCTGGCTCTCCATC-3', Tnfrsf18, forward 5'-CCTGCCAACCAGGCCAGAGGG-3', reverse 5'-GTCCAAAGTCTCCAGTCACCG-3'; and (E) Bcl6, forward 5'-CACACCCGTCCATCATTCAA-3', reverse 5'-TCTCCTCACCGTCCCTTTTT-3'. Data shown are representative of 3 identical experiments. The values represent mean $\pm \operatorname{SEM}(n=3)$. ns, $P>0.05$, Student's 1-tailed $t$ test.

In vivo specificity of nTreg-like autoantigen-specific iPSC-Tregs. To exert their suppressive effects, Tregs need to migrate to specific tissues or organs, and this requires Ag specificity (8). We and others have previously reported that Tregs are detected within inflamed tissues and transplanted grafts, suggesting that these Tregs could control effector $\mathrm{T}$ cells in the peripheral tissues at sites of ongoing immune responses (12-14). Tregs may block the pathogenic cells from filling up their appropriate niche by taking up the space. The Treg population was reduced in autoimmune prone animals and patients, and Tregs were defective in NOD mice (15). In this study, we observed that mice receiving autoantigen OVA-specific iPSC-Tregs maintained a high population of both $\mathrm{CD}^{+}$ and FoxP3 ${ }^{+}$cells in the pancreas, as compared with diabetic mice receiving control cells (iPSC-derived cells with empty vector) or nontissue-associated SM1-specific iPSC-Tregs by immunofluorescence examination (Figure $3 \mathrm{~A}$ ) and by flow cytometric analysis ( $90.1 \%$ vs. $12.5 \%$ or $16.8 \%$ at week 13 , Figure $3 \mathrm{~B})$. These results suggest that the autoantigen-specific iPSC-Tregs are organ/tissue-associated nTreg-like suppressive cells.

Autoantigen-specific iPSC-Tregs can prevent destruction of pancreatic $\beta$ cells and reduce autoimmune diabetes. Next, we injected diabetic mice with autoantigen-specific iPSC-Tregs. Two weeks after the cell transfer, blood glucose levels were measured. This cell transfer with autoantigen OVA-specific iPSC-Tregs but not control cells or nontissue-associated SM1-specific iPSC-Tregs significantly reduced blood glucose level with urine discharge in all of the diabetic mice $(P<0.0001$, Figure 4A and Supplemental Figure 3). T1D develops due to the destructive autoreactive immune response in which $\mathrm{CD} 8^{+} \mathrm{T}$ cells play a critical role. $\mathrm{CD} 8^{+} \mathrm{T}$ cells infiltrated into the pancreatic islets of the T1D patients at both initial and final destructive phases of autoimmune $\beta$ cells attack (16). The blood glucose level was increased because of the destruction of $\beta$ cells in the islets by pathogenic $\mathrm{CD}^{+} \mathrm{T}$ cells in the diabetic pancreas, which was evidenced in the above mouse diabetic model. More $\mathrm{CD} 8^{+} \mathrm{T}$ cells were accumulated in the pancreases of mice receiving control cells or nontissue-associated 
A

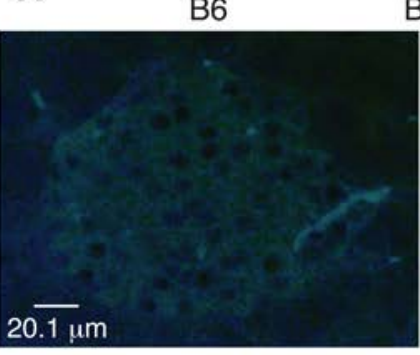

B6-mOVA x OT-I TCR double Tg

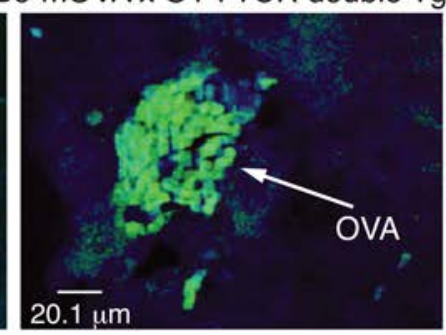

B

B6

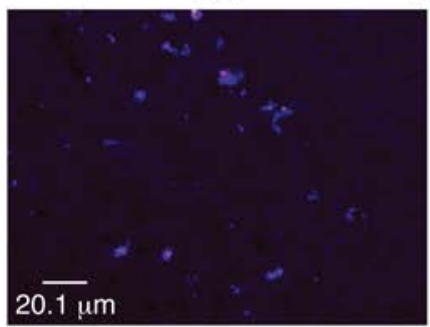

B6-mOVA x OT-I TCR double Tg

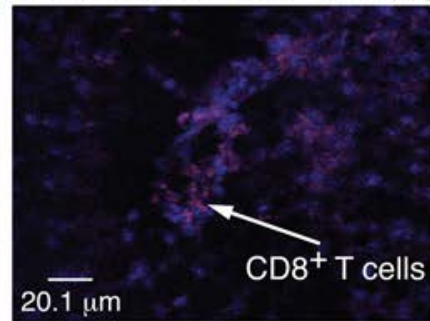

C

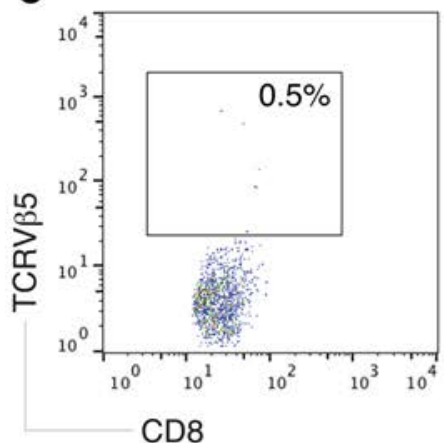

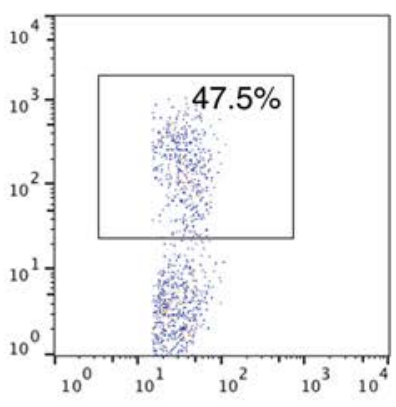

D
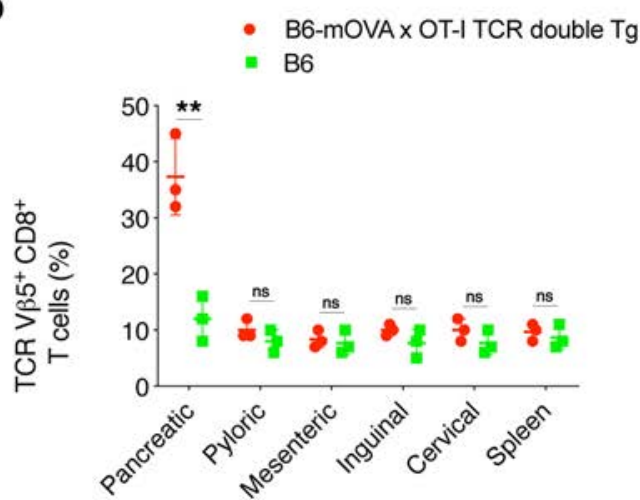

E

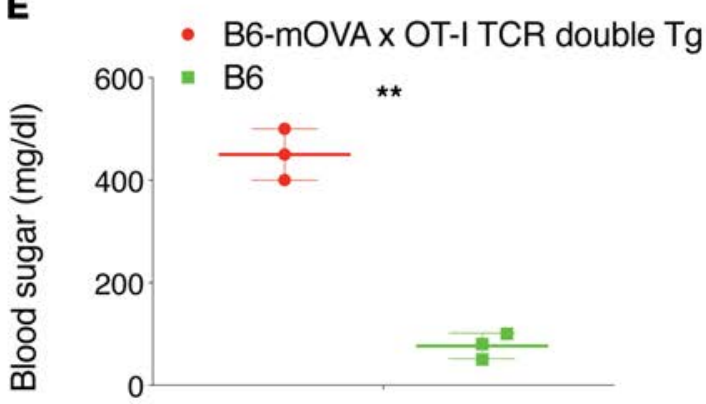

Figure 2. Accumulation of activated $\mathrm{CDB}^{+} \mathbf{T}$ cells in autoantigen-expressing pancreases in diabetic mice. Pancreases were isolated from B6-mOVA $\times$ OT-I TCR double-Tg mice and B6 mice at 9 weeks of age, including 1 week in which mice were challenged with vaccinia viruses expressing OVA (VACV-OVA). (A) Detection of OVA expression by immunohistochemically staining. OVA expression (arrow) is indicated (original magnification, $\times 200$ ). Data are representative of 5 mice per group in 3 independent experiments. Scale bar: $20.1 \mu \mathrm{m}$. (B) CD8 $8^{+}$T cell infiltration in the pancreas. CD8+ T cells (arrow) are indicated. Data are representative of 5 mice per group in 3 independent experiments. Scale bar: $20.1 \mu \mathrm{m}$. (C) OVA-specific CD8 ${ }^{+}$T cells in the pancreas. OVA-specific TCRV $\beta 5$ was analyzed by flow cytometry, after gating on $C D 8^{+}$populations from the pancreas. Data shown are representative of 3 independent experiments $(P<0.001$, Student's 1-tailed $t$ test). (D) Summarized analyses of OVA-specific CD8+ T cells in various locations. Data shown are representative of 5 mice per group in 3 independent experiments. Data shown are representative of 3 individual experiments. The values represent mean \pm SD. ${ }^{*} P<0.01 ;$ ns, $P>0.05$, multiple Student's 1-tailed $t$ test. (E) Blood glucose measurement. Data shown are representative of 3 individual experiments $(n=5)$. The values represent mean $\pm \mathrm{SD}$. ${ }^{* *} P<0.01$, Student's 1 -tailed $t$ test.

Ag-specific iPSC-Tregs than autoantigen-specific iPSC-Tregs by immunofluorescence examination (Figure 4B) and by flow cytometric analysis $(49.8 \%$ or $46.1 \%$ vs. $6.5 \%$, Figure 4 C). Only mice receiving autoantigen-specific iPSC-Tregs had a significantly reduced percentage of incidence of diabetes $(P<0.001$, Figure 4D). A large number of inflammatory cells had infiltrated into the pancreases of mice receiving control cells, whereas the infiltration was substantially reduced in mice receiving autoantigen-specific iPSC-Tregs, as shown by $\mathrm{H} \& \mathrm{E}$ staining (Figure $4 \mathrm{E}$ ). The total number of the islets was also reduced in mice receiving control cells as compared with autoantigen-specific iPSC-Tregs $(P<0.01$ or $P<0.001$, Figure $4 \mathrm{~F})$. To detect the destruction of $\beta$ cells in the islets of diabetic mice, we stained pancreases with insulin. The islets were reduced in size and number in mice receiving control cells, whereas mice receiving autoantigen-specific iPSC-Tregs were protected from the destruction, by immunofluorescence examination (Figure $4 \mathrm{G}$ ). The islets were partially destructed by pathogenic $\mathrm{CD} 8^{+} \mathrm{T}$ cells, which caused the increase of blood sugar and developed autoimmune diabetes, but some insulin ${ }^{+}$staining in diabetic mice was still present. Collectively, these results indicate that autoantigen-specific iPSC-Tregs are specific and effective in protecting the hosts from islet destruction and in promoting insulin secretion to prevent the mice from diabetes mellitus. 

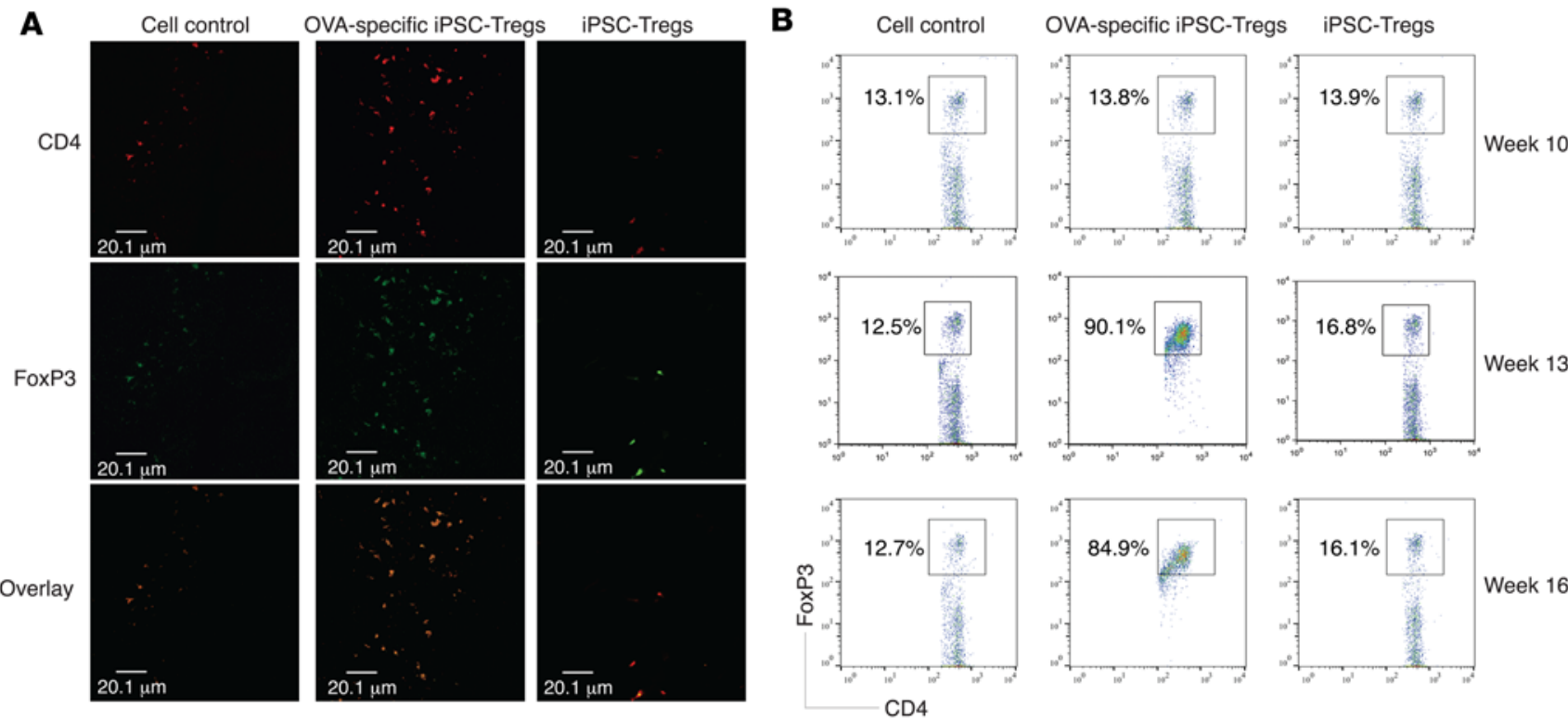

Figure 3. Accumulation of autoantigen-specific iPSC-Tregs in the pancreases of diabetic mice following adoptive transfer. B6-mOVA Tg $\times$ OT-I TCR double-Tg mice were immunized with VACV-OVA. At week 10, control cells or OVA- or SM1-specific pre-iPSC-Tregs were transferred into diabetic mice. Before or after the cell transfer, mice were sacrificed and their pancreases were isolated for analysis of CD4 and FoxP3. (A) Immunohistology at week 13 (original magnification, $\times 200$ ). Scale bar: $20.1 \mu \mathrm{m}$. (B) Flow cytometric analysis at week 10 (before the cell transfer), 13, and 16. Data are representative of 5 mice per group in 3 independent experiments.

Autoantigen-specific iPSC-Tregs can decrease the expression of ICAM-1. A number of mononuclear cells, including macrophages and $\mathrm{CD} 4^{+} \mathrm{T}$ cells, were found to be initially predominant in the autoimmune process taking place in T1D $(17,18)$. In autoimmune diabetes, these cells played an important role in the early infiltrating process and were shown to express ICAM-1, which served to bind to lymphocytes and possibly to monocytes and polymorphonuclear leukocytes (19). The infiltrated ICAM-1 can promote the release of several cytokines that modulate the expression of the adhesion molecules, thus increasing the adhesion of leukocytes and other autoreactive $\mathrm{T}$ cells (20). These autoreactive $\mathrm{T}$ cells, after migrating into the pancreas, start to destroy $\beta$ cells. We thus examined the expression of ICAM-1 in the pancreases. The high expression of ICAM-1 in the pancreases of the diabetic mice, which was undetectable in the normal pancreases, was significantly reduced in diabetic mice receiving autoantigen-specific iPSC-Tregs but not other control cells (Figure 5, A and B). In addition, the high expression of ICAM-1 on the effector $\mathrm{CD}^{+} \mathrm{T}$ cells in the pancreases of the diabetic mice receiving control cells $(84.4 \%$ or $78.9 \%)$, which was undetectable in the normal pancreases $(0.35 \%)$, was markedly reduced in those of mice receiving autoantigen-specific iPSC-Tregs (18.4\%) (Figure 5C). In addition, the expression of ICAM-1 on CD11 ${ }^{+}$ cells in the pancreases of diabetic mice receiving control cells $(15.64 \%$ or $12.83 \%)$, which was at a low level in the normal pancreases $(4.89 \%)$, was considerably reduced in those of mice receiving autoantigen-specific iPSC-Tregs (5.68\%) (Figure 5D). Because CD11b is expressed on the surface of various leukocytes, including monocytes/macrophages, granulocytes, neutrophils, NK cells, and dendritic cells, the reduction of $\mathrm{CD} 11 \mathrm{~b}^{+} \mathrm{ICAM}-1^{+}$cells indicated the inhibition of leukocyte adhesion and migration to mediate the inflammatory response. Taken together, these results suggest that the autoantigen-specific iPSC-Tregs can reduce the expression of ICAM- 1 in diabetic mice, inhibit the migration of autoreactive $\mathrm{CD}^{+} \mathrm{T}$ cells into the pancreas, and, ultimately, protect the pancreatic $\beta$ cells from destruction.

Autoantigen-specific iPSC-Tregs can migrate to the pancreas and produce a large amount of suppressive cytokines. In BDC2.5/NOD mice, FoxP3 was highly expressed in insulitic lesions where the function was impaired due to alterations in their gene expression profile $(21,22)$. We previously showed that the iPSC-Tregs exhibited a similar expression of Treg signature genes and produced IL-10 and TGF- $\beta$ (12). In the current study, we observed very few numbers of FoxP3-expressing $\mathrm{T}$ cells in the pancreases of diabetic mice, and these cells did not secret IL-10 and TGF- $\beta$, which are the two key cytokines that are the hallmark for functional Tregs and secreted by activated and functional Tregs. 
A

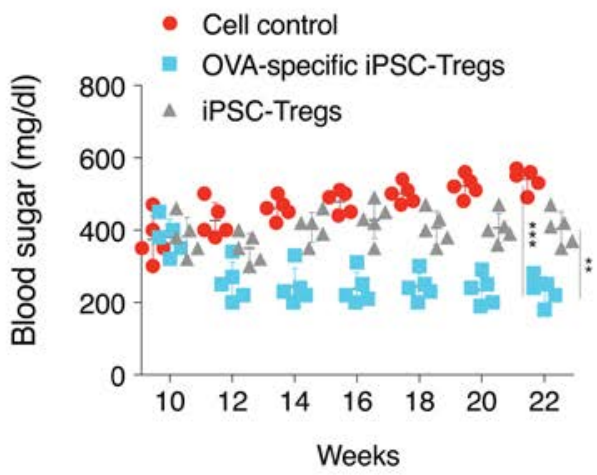

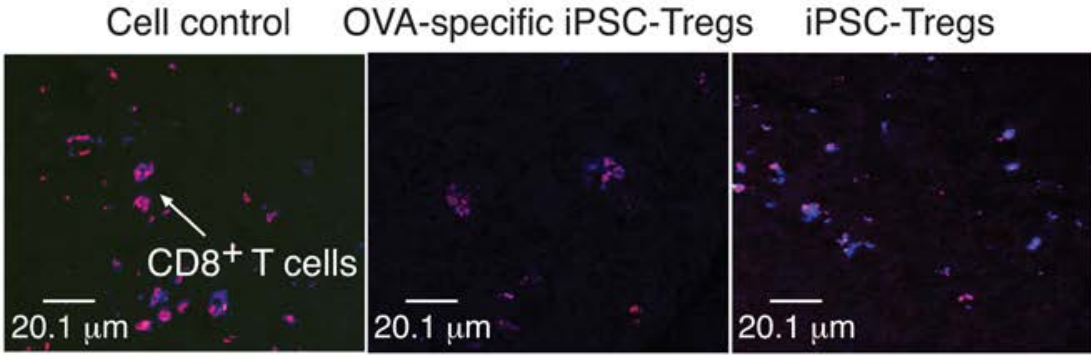

C
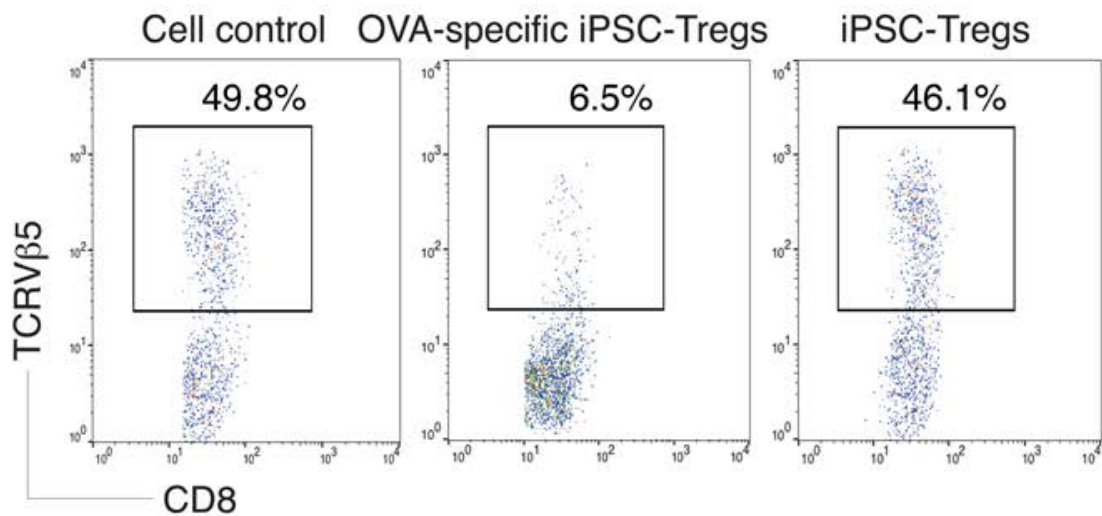

D

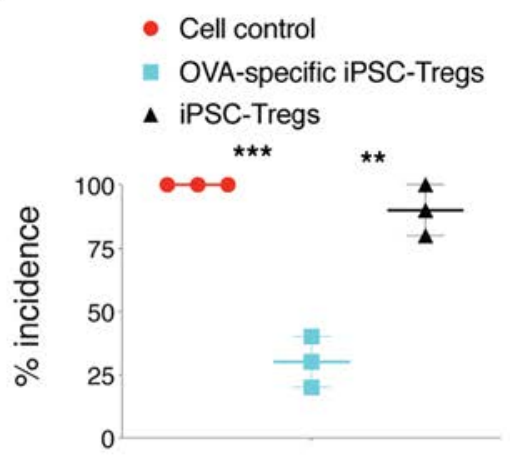

$\mathbf{E}$

Cell control OVA-specific iPSC-Tregs iPSC-Tregs
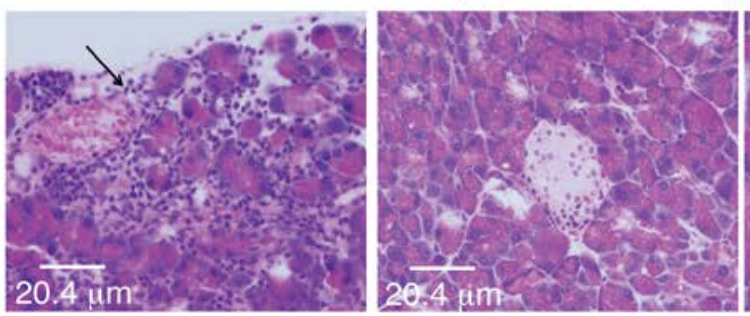

a

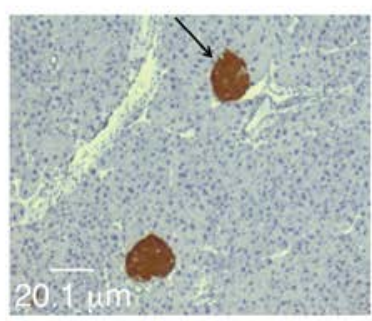

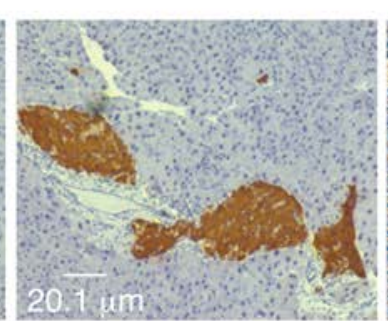
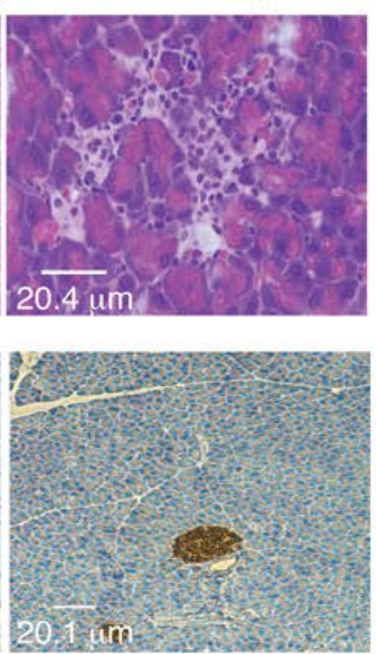

$\mathbf{F}$

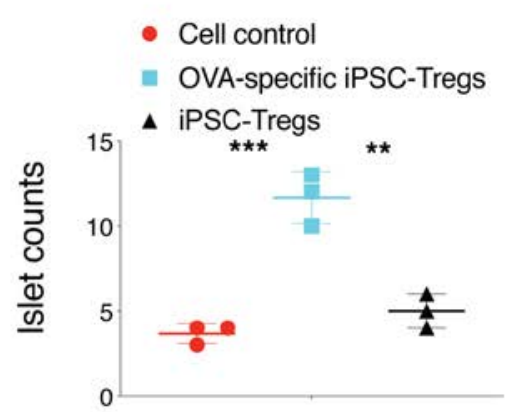

Figure 4. Prevention of destruction of pancreatic $\beta$ cells and suppression of autoimmune diabetes by autoantigen-specific iPSCs-Tregs. Control cells or pre-iPSC-Tregs were adoptively transferred into diabetic mice, as described in Figure 3. (A) Blood sugar measurement at various weeks. Data shown are representative of 5 mice per group in 3 independent experiments. ${ }^{* *} P<0.001$; ${ }^{* *} P<0.0001$, 2-way ANOVA analysis. (B) Immunofluorescence detection of pathogenic immune cells. Mice were sacrificed and pancreases were isolated for immunohistochemistry staining with CD8 ${ }^{+} T$ cells (original magnification, $\times 200$ ). Scale bar: $20.1 \mu \mathrm{m}$. Data are representative of 5 mice per group in 3 independent experiments. (C) Flow cytometric analysis of pathogenic immune cells. Pancreatic lymph nodes (LNs) were isolated, and single-cell suspensions were prepared for OVA-specific CD8 ${ }^{+} \mathrm{TCRV} 5^{+}$staining and analyzed by flow cytometry. Data shown are representative of 3 identical experiments $(P<0.001$, Student's 1-tailed $t$ test). (D) Percentage of incidence of diabetes at week 22. Data shown are representative of 5 mice per group in 3 independent experiments. The values represent mean \pm SD. ${ }^{* *} P<0.001$; ${ }^{* * *} P$ $<0.001,1$-way ANOVA analysis. (E) Representative photomicrographs (H\&E staining) of the islet inflammation (original magnification, $\times 200$ ). Scale bar: $20.4 \mu \mathrm{m}$. Cellular infiltrations (arrow) are indicated. Data are representative of 5 mice per group in 3 independent experiments. (F) Islet count from sections of 5 individual pancreases in each group. Data are represented as the mean \pm SD of 3 independent experiments ( ${ }^{* * *} P<0.001,1$-way ANOVA analysis). (G) Representative photomicrographs (immunofluorescence staining) of islet destruction (original magnification, $\times 200$ ). Scale bar: $20.1 \mu \mathrm{m}$. Insulin-producing cells (arrow) are indicated. Data are representative of 5 mice per group in 3 independent experiments. 
$\mathbf{A}_{\mathrm{N}}$

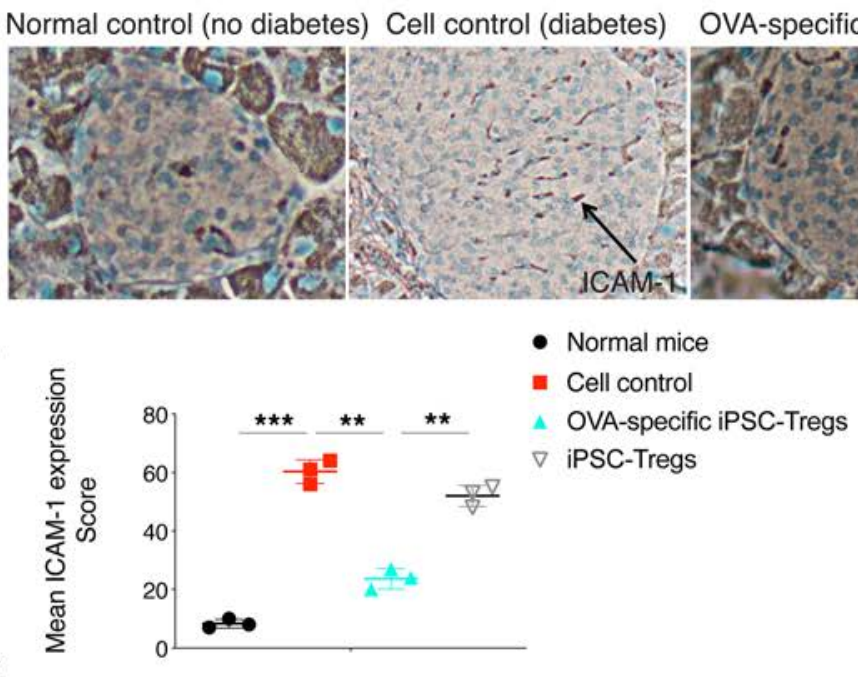

Normal control (no diabetes) Cell control (diabetes) OVA-specific iPSC-Tregs
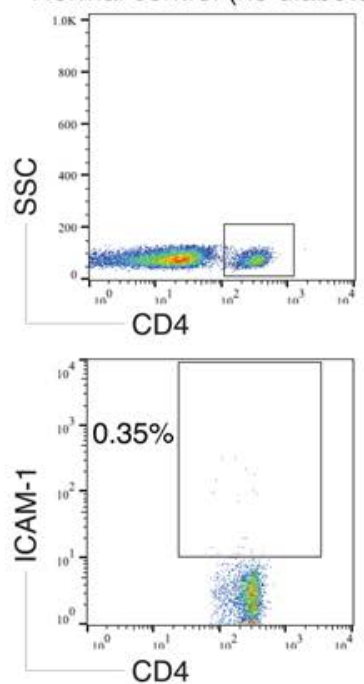

D

Normal control (no diabetes)

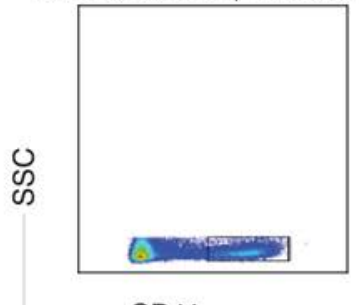

CD11

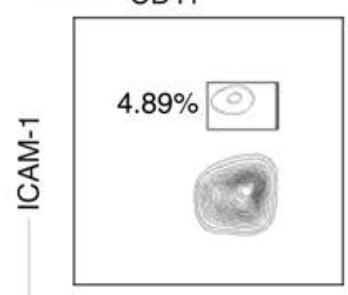

CD11
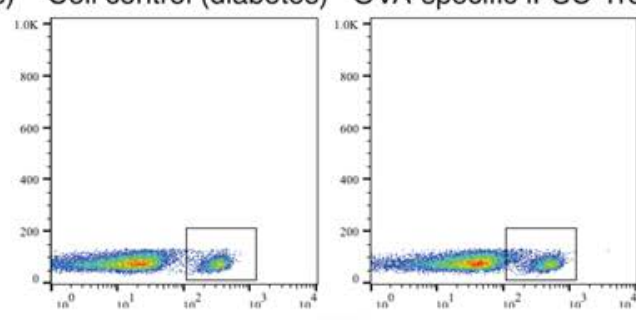

$\downarrow$
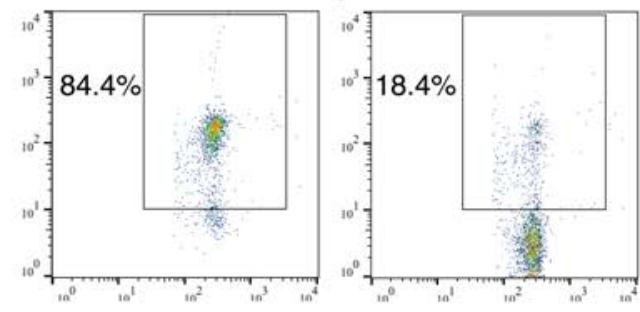

Cell control (diabetes)
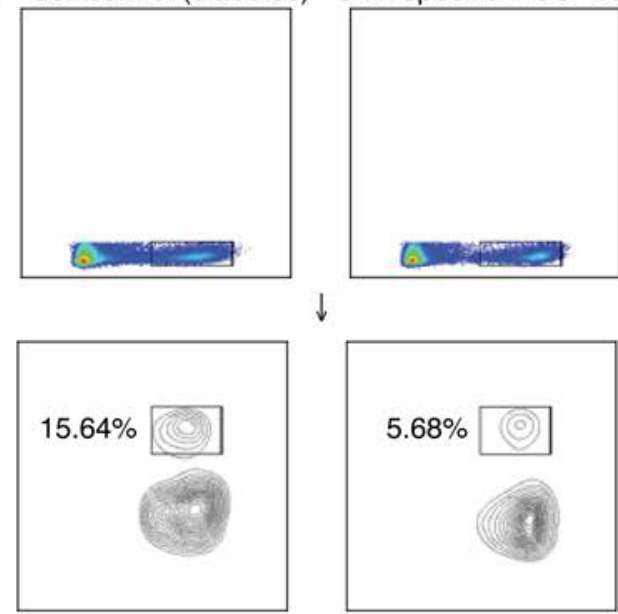

iPSC-Tregs
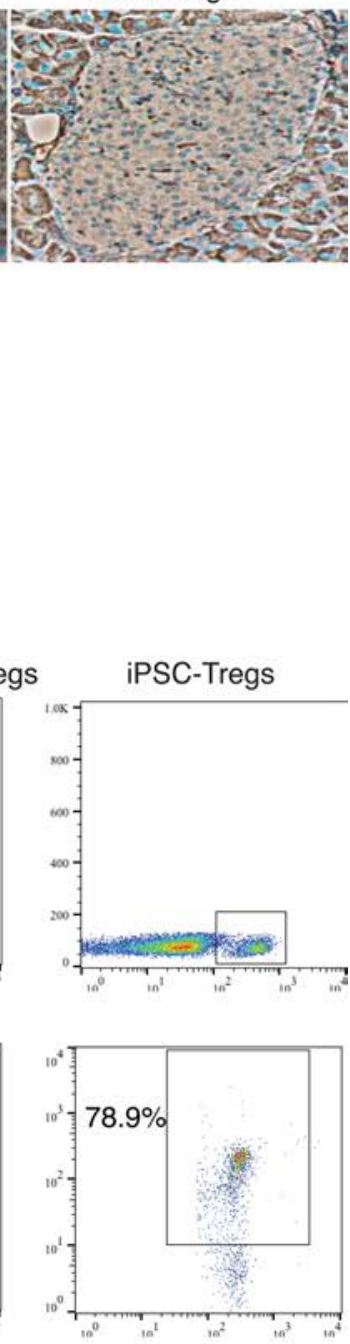

OVA-specific iPSC-Tregs
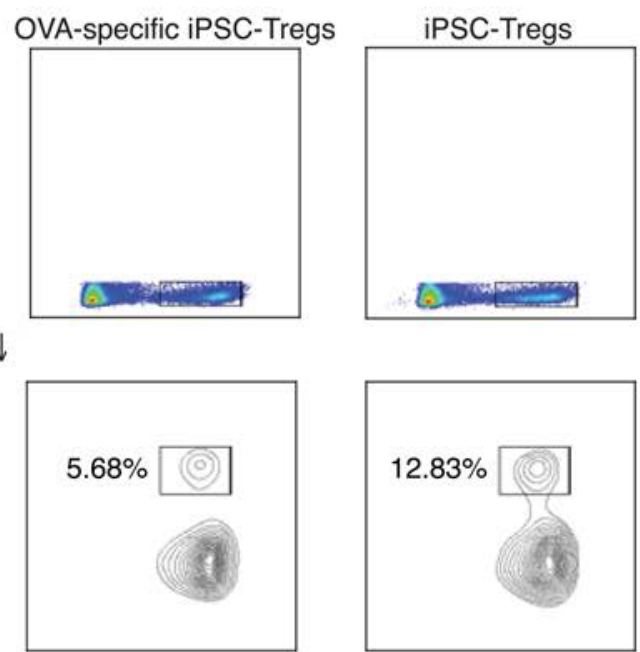

Figure 5. Reduction of ICAM-1 expression in the diabetic pancreas by autoantigen-specific iPSCTregs. Mice were sacrificed 4 weeks after adoptive cell transfer, and the pancreases were isolated from nondiabetic, diabetic (cell transfer control), and diabetic (Ag-specific iPSC-Treg transfer) mice. Samples were prepared for immunohistochemistry staining. (A) ICAM-1+ zones (arrow) are indicated (original magnification, $\times 200$ ). Data are representative of 5 mice per group in 3 independent experiments. (B) Quantification of ICAM-1 expression from sections of 5 individual pancreases in each group. Data are represented as the mean $\pm \mathrm{SD}$ from 3 independent experiments ( ${ }^{*} P<0.01 ;{ }^{* *} P<0.001$, 1-way ANOVA). (C and D) Flow cytometry analysis of ICAM-1 expression in pancreatic $C D 4^{+} T$ or $C D 11 b^{+}$ cells. The pancreatic lymph nodes (LNs) were isolated, and single-cell suspensions were prepared for ICAM-1 staining on $\mathrm{CD}^{+}$or $\mathrm{CD} 11 \mathrm{~b}^{+}$ cells and analyzed by flow cytometry. Data shown are representative of 3 identical experiments.

We further showed that the pancreatic $\mathrm{CD} 4^{+} \mathrm{T}$ cells from mice receiving autoantigen-specific iPSCTregs produced substantially more IL-10 (66.1\% vs. $12.9 \%$ or $15.1 \%)$ and TGF- $\beta(5.61 \%$ vs. $0.62 \%$ or $1.68 \%$ ), as compared with those from mice receiving control cells (Figure 6). These results support the assumption that autoantigen-specific iPSC-Tregs accumulating in the pancreas can secrete large amounts 
of suppressive cytokines (IL-10 and TGF- $\beta$ ), reduce the expression of ICAM-1, and prevent the migration of pathogenic $\mathrm{CD}^{+} \mathrm{T}$ cells into the pancreas, thus protecting the pancreas from destruction.

Autoantigen-specific iPSC-Tregs can decrease the production of IFN- $\gamma$ by pathogenic immune cells in the diabetic pancreatic islets. Treatment of NOD mice with an anti-IFN- $\gamma$ monoclonal antibody could block diabetes (23); conversely, overproduction of IFN- $\gamma$ in pancreatic islets provoked the disease (24). We next determined the secretion of IFN- $\gamma$ in the diabetic islet. We found more IFN- $\gamma$-producing pancreatic CD 8 and CD4 T cells in mice receiving control cells ( $31.4 \%$ and $30.4 \%$ or $29.7 \%$ and $28.6 \%$ ); by contrast, both of the IFN- $\gamma$-producing pancreatic CD8 and CD4 T cells were significantly reduced in mice receiving autoantigen-specific iPSC-Tregs (14.6\% and 10.9\%) (Figure 7). These results indicate that the autoantigen-specific iPSC-Tregs can protect the islets from destruction by suppressing the secretion of IFN- $\gamma$ produced by the pathogenic immune cells.

\section{Discussion}

We and others have previously reported that adoptive transfer of stem cells has or stem cell-derived Tregs has the ability to suppress the development of autoimmunity in various animal models $(11,12,25,26)$. However, the suppressive mechanisms behind this suppression remain to be fully defined. By investigating the adoptive transfer of autoantigen-specific iPSC-Tregs in a murine model of T1D, we demonstrate that adoptive transfer significantly reduced the high ratio of $\mathrm{CD} 8^{+}$to $\mathrm{CD} 4^{+} \mathrm{T}$ cells in the pancreases of diabetic mice. We also show a critical role of this transfer in reducing the expression of ICAM-1 in inflamed pancreatic tissues and preventing the accumulation of pathogenic $\mathrm{CD} 8^{+} \mathrm{T}$ cells in the pancreases and diabetic pathogenesis. We further demonstrate that autoantigen-specific iPSC-Tregs associated with inflamed tissue and protected the islets from destruction through suppressing the production of proinflammatory cytokine IFN- $\gamma$. These findings may help us better understand the pathogenesis of autoimmunity in T1D and provide a foundation for therapeutic use of the stem cell-derived tissue-associated Tregs in the treatment of T1D (27).

Among animal models of autoimmune diabetes, the NOD mouse is a widely used animal of autoimmune T cell-mediated T1D (spontaneously nonobese diabetic). The NOD mouse model clearly shows the leukocytic infiltration into the pancreatic islets (insulitis) and autoimmune destruction of the pancreatic $\beta$ cell in female mice (2-4 weeks), which occurs later in male mice (5-7 weeks). Insulitis in NOD mice shows a combination of $\mathrm{T}$ cells (both $\mathrm{CD}^{+}$and $\mathrm{CD}^{+}$), B cells (28), and inconstant numbers of macrophages/ dendritic cells. In addition, a dominant-negative mutation in the mouse insulin 2 gene (Ins $2^{\text {Akita }}$ ) produces a severe insulin deficiency syndrome exclusive of autoimmune participation and various transgenes overexpressed in $\beta$ cells. Moreover, pharmacologically induced T1D (without autoimmunity) by alloxan or streptozotocin produces hyperglycemia in most strains of mice. Several low doses of streptozotocin combing direct $\beta$ cell toxicity with local inflammation also elicit T1D in a male sex-specific fashion (29). To determine the suppressive mechanisms of stem cell-derived tissue-associated Tregs, we employed a mouse model of T1D by crossing B6-mOVA Tg mice with OT-I TCR Tg mice. Around 8 weeks of age, blood glucose levels were measured in $\mathrm{F}_{1}$ mice. Approximately $20 \%-40 \%$ of the mice were found to be diabetic; one potential explanation for this occurrence is that the OT-I CD8 ${ }^{+} \mathrm{T}$ cells may be tolerated during $\mathrm{T}$ cell development in the thymuses of $F_{1}$ mice. As we propose in our model in which mice will develop autoimmune diabetes due to a large amount and the autoreactivity of $\mathrm{CD} 8^{+} \mathrm{T}$ cells, we activated tissue-associated $\mathrm{CD}^{+} \mathrm{T}$ cells by inoculating VACV-OVA into the mice in which the viruses-induced $\mathrm{CD}^{+} \mathrm{T}$ cells became highly polyfunctional (30). All mice developed diabetes with high blood glucose levels (Supplemental Figure 3) and more urine discharge, for which mice needed additional animal care. In this mouse model of T1D, OVA protein serves as inflamed tissue-associated autoantigen and is highly expressed in the pancreas. OVA-specific $\mathrm{CD} 8^{+} \mathrm{T}$ cells act as central pathogenic immune cells that accumulate in the pancreas and cause pancreatic $\beta$ cell destruction and T1D (Figure 2). In addition, in this mouse model of T1D, we observed a non-OVA-specific $\mathrm{CD} 4^{+}$population existing in the pancreas (Figure 3 ), and these $\mathrm{CD} 4^{+} \mathrm{T}$ cells produced IFN- $\gamma$ (Figure 7), which might also involve to the disease pathogenesis. These FoxP3-CD4 ${ }^{+} \mathrm{T}$ cells in the pancreas were TCRV $\alpha 2^{-} / \mathrm{V} \beta 5^{-}$, indicating that $\mathrm{T}$ cell activation was not through OVA-specific TCR. Of note, the number of these effector $C D 4^{+} \mathrm{T}$ cells was dramatically reduced in mice receiving OVA-specific but not SM1-specific iPSC-Tregs (Supplemental Figure 4). These results also indicate that tissue-associated (OVA-specific) iPSC-Tregs are more effective than nontissue-associated (SM1-specific) iPSC-Tregs. Because of the challenge of VACV-OVA, these non-OVA-specific CD4 ${ }^{+} \mathrm{T}$ cells mainly originating from the RIP-mOVA descent may undergo bystander activation $(31,32)$. However, addition studies are needed on the pathways leading to bystander $\mathrm{T}$ cell activation under the condition. 

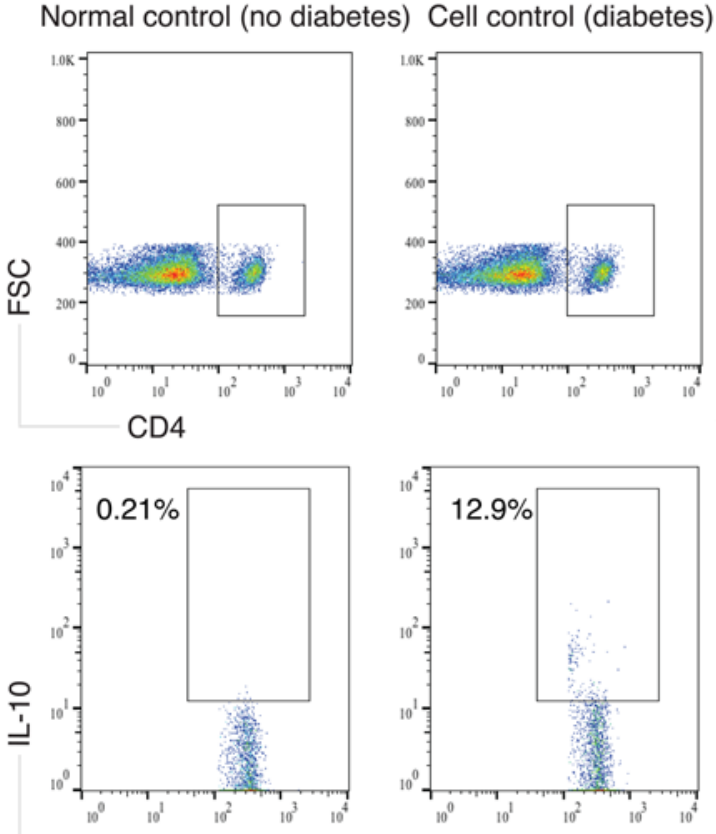

CD4

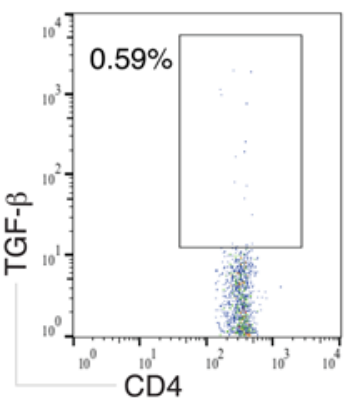

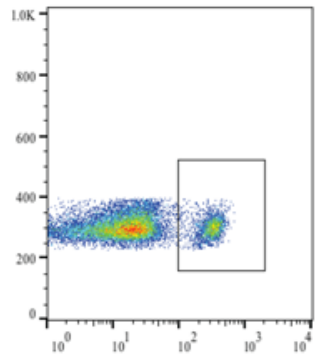
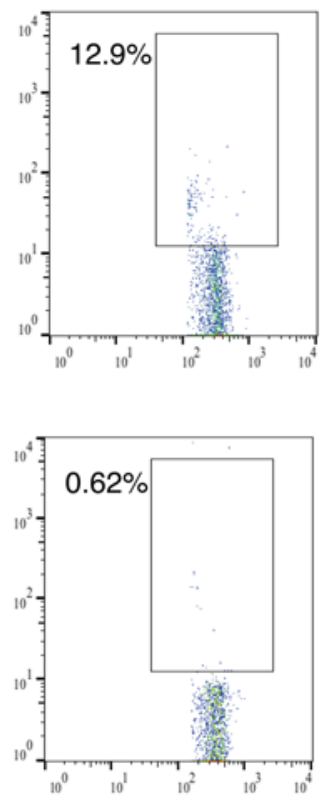

OVA-specific iPSC-Tregs

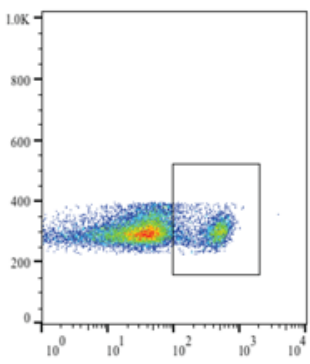

$\downarrow$

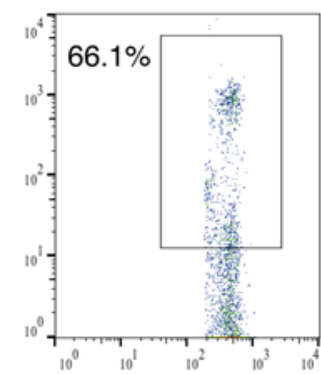

iPSC-Tregs
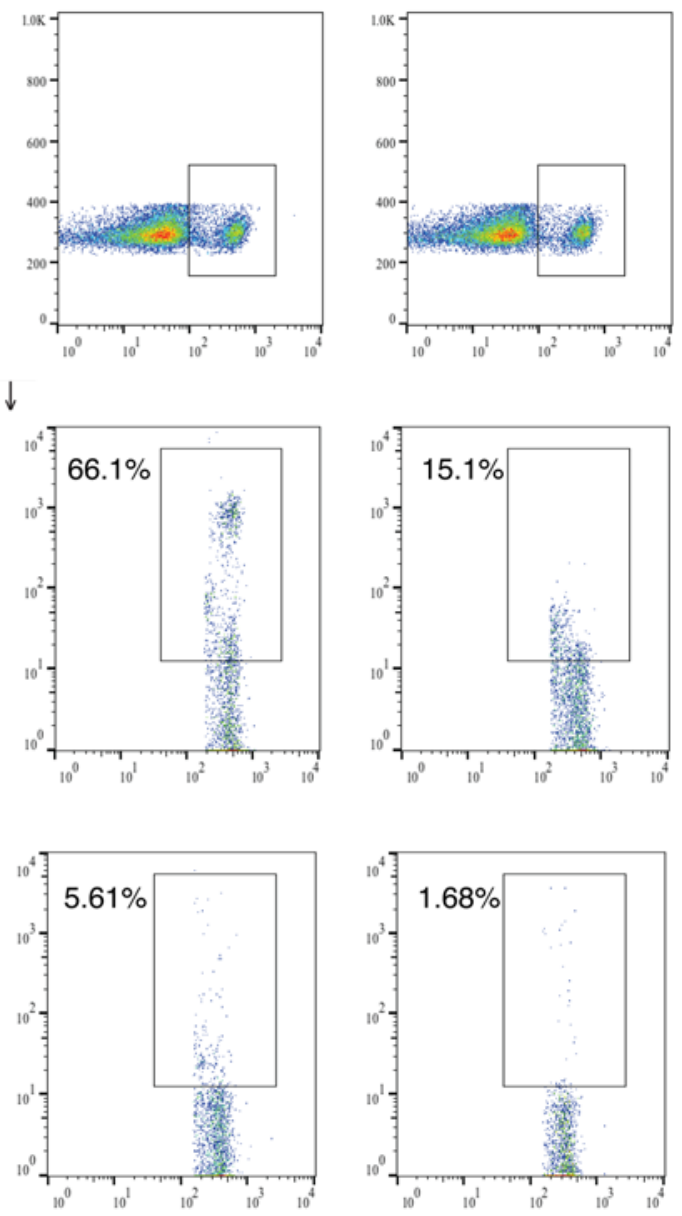

Figure 6. Induction of IL-10 and TGF- $\beta$ by autoantigen-specific iPSC-Tregs. Mice were sacrificed 4 weeks after adoptive cell transfer, and single-cell suspension was prepared from the pancreatic lymph nodes (LNs). Cells were stimulated with plate-coated CD3 and soluble CD28 antibodies and then stained with CD4 TCF- $\beta$, and IL-10. The CD4 population was gated, and the production of TCF- $\beta$ and IL-10 was analyzed. Data shown are representative of 3 identical experiments.

Tregs are an important component of self-tolerance, and numerous studies have demonstrated the effects of Tregs on natural and induced autoimmune diseases in various mouse models $(33,34)$. Targeting Tregs as a treatment for autoimmune disorders is an attractive approach, as there is an emerging consensus that many patients suffering from autoimmune disease have dysfunctional Tregs $(35,36)$. Given the manageability of murine models, the evidence for Tregs in control of autoimmune diseases has recently become clearer, particularly as a therapeutic intervention. In well-established models of rheumatoid arthritis (37), multiple sclerosis (38), and systemic lupus erythematosus (39), adoptive transfer of polyclonal Tregs could prevent or slow disease progression when administered prior to disease occurrence. In multiple sclerosis, Ag-specific Tregs targeting the disease-associated Ags were highly efficacious in reverting the ongoing disease $(8,40)$. Specifically, the nonobese T1D NOD.Cd28-1mouse model deficient in Tregs developed diabetes at an accelerated rate as compared with NOD mice. Injection of NOD Tregs into NOD.Cd $28^{-1-}$ mice could delay and, in some cases, prevent the development of diabetes (41). In the current study, we have confirmed that adoptive transfer of stem cell-derived autoantigen-specific Tregs but not control cells, nonspecific iPSC-Tregs, or nontissue-associated Ag-specific iPSC-Tregs caused a high level of accumulation of these cells in the pancreas and reversed the disease process of autoimmune diabetes in the mouse model of T1D that was caused by pathogenic CD8 ${ }^{+} \mathrm{T}$ cells (Figure 4 and Supplemental Figure 3). A small number of Ag-specific iPSCTregs was observed in LNs and spleen (Supplemental Figure 5). A possible working mechanism of stem cell-derived autoantigen-specific Tregs is mediated through the secretion of large amounts of suppressive cytokines (e.g., IL-10 and TGF- $\beta$ ) that were identified (Figure 6). Of note, Ag-specific iPSC-Tregs have a functional stability for which we had previously demonstrated in an autoimmune condition (12), and FoxP3 expression in these cells persisted for 6 weeks after adoptive transfer in the diabetic mice (Figure 3). 
A Normal control (no diabete
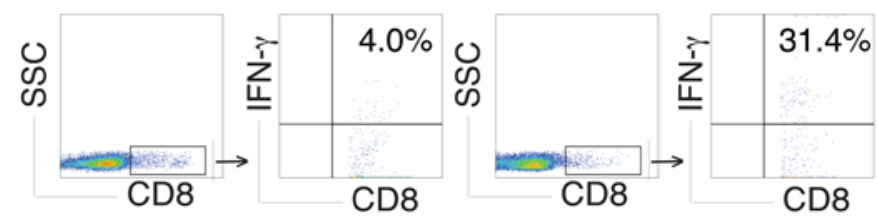

Cell control (diabetes)

OVA-specific iPSC-Tregs
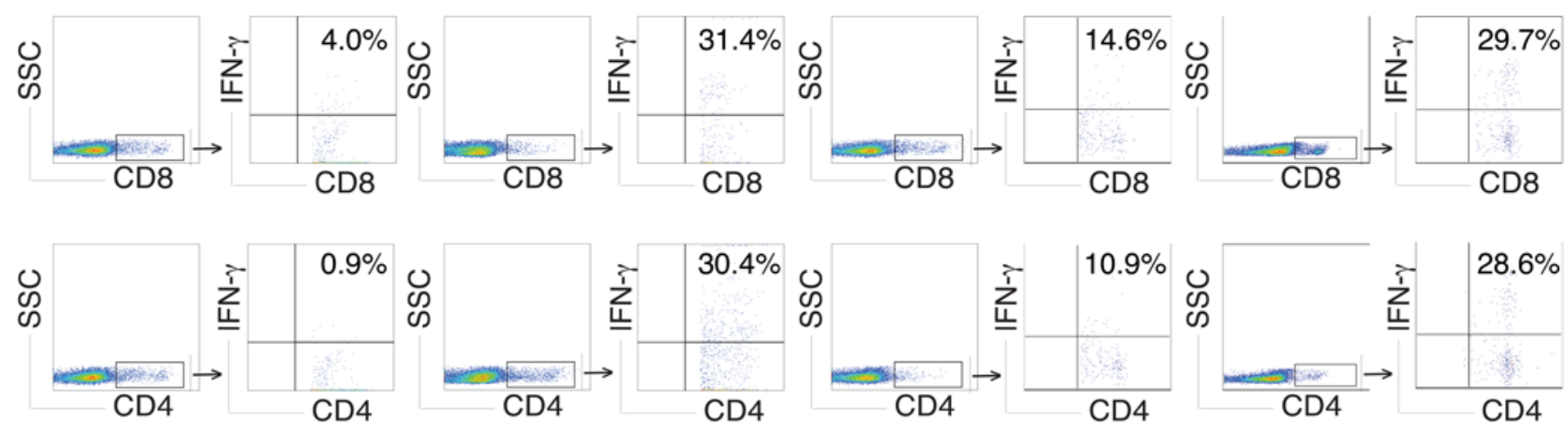

B
- Normal mouse control
- Cell control
$\triangle$ OVA-specific iPSC-Tregs
$\nabla$ iPSC-Tregs
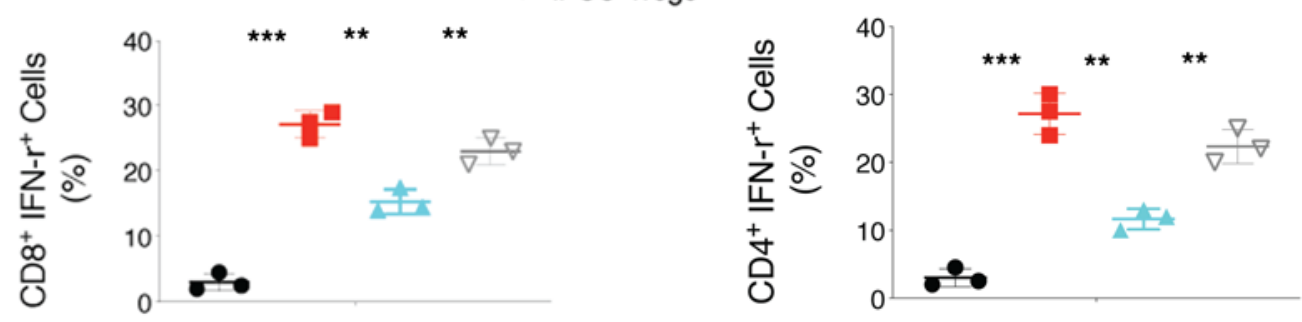

Figure 7. Downregulation of IFN- $\gamma$ by autoantigen-specific iPSC-Tregs in the diabetic pancreatic islets. Mice were sacrificed 4 weeks after adoptive cell transfer, and single cell suspension was prepared from the pancreatic lymph nodes (LNs). Cells were stimulated with plate-coated CD3 and soluble CD28 antibodies, and then stained with CD4, CD8, and IFN- $\gamma$ for flow cytometric analysis. (A) IFN- $\gamma$ production in CD4 ${ }^{+}$or $C D 8^{+}$population. Data shown are the representative of 3 identical experiments. (B) Quantification of IFN- $\gamma$ production. Data shown are representative of 3 identical experiments $(* * P<0.01$; $* * * P<0.001,1$-way ANOVA).

We further show that ICAM-1 (also known as CD54), a member of the Ig-like superfamily of adhesion proteins, plays a critical role in the accumulation of the pathogenic $\mathrm{CD} 8^{+} \mathrm{T}$ cells in the pancreases of the mice with autoimmune diabetes. ICAM-1 is a cell surface glycoprotein expressed in endothelial cells and some immune cells. ICAM-1 expressed in diabetic pancreatic tissues can bind to integrins of type CD11a/CD18 or CD11b/CD18 on pathogenic CD8 ${ }^{+} \mathrm{T}$ cells, and this binding facilitates the accumulation of pathogenic $\mathrm{CD}^{+} \mathrm{T}$ cells in the panaceas. The expression of ICAM-1 in the pancreases of diabetic mice is much higher than that in normal mice. In contrast, in the pancreases of diabetic mice, the adoptive transfer of autoantigen-specific iPSC-Tregs can dramatically reduce the expression of ICAM-1. This cell transfer can also reduce the accumulation of autoreactive $\mathrm{CD} 8^{+} \mathrm{T}$ cells in the pancreases and eventually protect the pancreatic $\beta$ cells from further devastation (Figure 5). This conclusion is in line with those of previous studies showing that the expression of ICAM-1 is critical for the development of experimental autoimmune encephalomyelitis $(42,43)$.

The role of IFN- $\gamma$, a prototypical Th1 cytokine, has been appreciated in the development of T1D, because the treatment of NOD mice with an anti-IFN- $\gamma$ monoclonal antibody prevented the development of the autoimmune diabetes $(23,24)$. We detected an elevated amount of IFN- $\gamma$ in both effector CD8 and CD4 T cells of the diabetic islets and showed that adoptive transfer of stem cell-derived autoantigen-specific Tregs markedly decreased the production of the proinflammatory cytokine in the pathogenic $\mathrm{T}$ cells. We demonstrated that the autoantigen-specific iPSC-Tregs protected the islets from mass destruction via suppressing the production of pathogenic IFN- $\gamma$ (Figure 7). As other proinflammatory cytokines, such as IL-21 (44), IL-17 (45), TNF- $\alpha$ (46), and IL-1 $\beta$ (47) also play roles in the pathogenesis of T1D, studies on these proinflammatory cytokines would be needed to better exploit the stem cell-derived tissue-associated Tregs as a therapeutic approach against autoimmune disorders. 
The Tg model used in this study highly expressed OVA as surrogate autoantigen and received approximately 2 million of OVA-specific iPSC-Tregs for the treatment, which may be much higher than in the clinical setting with diverse low-abundance target peptides. However, the understanding of islet Ags has previously resulted in translation into new strategies by targeting tissue-specific immune interventions to prevent disease progression as well as to reverse T1D. A big advantage of our approach is the development of large numbers of autoantigen-specific nTreg-like suppressive cells (Figure 1 and Supplemental Figures 1 and 2), which can accumulate in the inflamed tissues and suppress local pathogenic immune cells. In addition, the stem cellderived Tregs maintain stability for up to 3 months $(11,12)$, because the overexpression of FoxP3 has the ability to suppress the potential switch of Tregs to Th17 cells. Of note, the selection of optimal autoantigens for the development of tissue-associated Tregs is critical for the success of the Treg-based T1D immunotherapy.

Taken together, the current study provides insight into the mechanisms by which stem cell-derived tissue-associated Tregs suppress autoimmunity in T1D and suggests that stem cell-derived autoantigen-specific Tregs have a great potential to be adapted as an immunotherapy for T1D.

\section{Methods}

Cell lines and mice. The mouse iPS-MEF-Ng-20D-17 cell line was obtained from the RIKEN Cell Bank (48). The OP9-DL1/DL4/I-A ${ }^{\mathrm{b}}$ cell line was generated by a retroviral transduction of the OP9 cells (12). The SNL76/7 cell line (ATCC SCRC-1049) was purchased from ATCC. C57BL/6 (B6), Rag1 $1^{-/-}$, B6 mOVA RIP (RIP-mOVA) Tg, and OT-I TCR Tg mice were purchased from The Jackson Laboratory.

Cell culture. iPSCs were maintained on feeder layers of irradiated SNL76/7 cells in 6-well culture plates (Nunc) and were passaged every 3 days (48).

Retroviral transduction and generation of OVA-specific iPSC-Tregs. cDNA for FoxP3 with OVA 322-339 (ISQAVHAAHAEINEAGR)-specific I-A ${ }^{b}$-restricted TCR genes $(\mathrm{V} \alpha 2$ and V $\beta 5$; obtained from Dario A. Vignali, University of Pittsburgh, Pittsburgh, Pennsylvania, USA) or LCMV (SMARTA1; SM1) gp61 (GLKGPDIYKGVYQFKSVEFD)-specific I-A ${ }^{\mathrm{b}}$-restricted TCR genes (V $\alpha 2$ and V $\beta 8$; obtained from Matthew A. Williams, University of Utah, Salt Lake City, Utah, USA) was used for retroviral transduction of mouse iPSCs and the generation of OVA- or SM1-specific iPSC-Tregs (12).

Antibodies. PE-, PE/Cy7-, Alexa Fluor 647-, APC-, or APC/Cy7-conjugated anti-mouse TCRV $\beta 5$ (MR9-4), CD4 (GK1.5), CD11b (M1/70), TGF-31 (TW7-16B4), and FoxP3 (MF-14) were obtained from Biolegend. FITC- or PE-conjugated anti-mouse CD8 (6A242) was obtained from Santa Cruz Biotechnology. APC-conjugated IL-10 (JES5-16E3) was purchased from BD Biosciences. Rabbit insulin antibody (C27C9; 3014) was purchased from Cell Signaling and anti-ICAM1 (YN1/1.7.4; ab25375) antibody was obtained from Abcam.

Flow cytometric analysis. T cells from the pancreatic LNs were collected and intracellular IL-10 and TGF- $\beta$ were analyzed by flow cytometry after gating on live $\mathrm{CD} 4^{+}$or $\mathrm{CD} 8^{+}$cells.

$R T$-PCR. MiDR OTII 2A FoxP3-transduced mouse iPSCs were cocultured with OP9-DL1-DL4-I-A in the presence of $\mathrm{mFlt}-3 \mathrm{~L}$ for 7 days. Differentiated iPSCs were separated and adoptively transferred into Rag1 $1^{-/-}$mice. Mice were housed for 6 weeks for in vivo maturation of the iPSC-Tregs. Six weeks later, spleens and LNs were collected from normal B6 mice and cell transferred Rag $1^{-/}$mice. CD $4^{+} \mathrm{CD} 25^{+}$ cells were sorted and total RNA was extracted from sorted cells using QIAgen RNeasy mini kits. Samples were subjected to reverse transcription using a high-capacity cDNA synthesis kit (Applied Biosystems). PCR analysis was performed by TaqMan real-time PCR (Thermo Fisher Scientific).

Ex vivo stimulation assay. Mice were sacrificed 4 weeks after adoptive cell transfer and single-cell suspension was then prepared from the pancreatic LNs. Cells were stimulated with plate-coated CD3 plus soluble CD28 antibodies. Productions of IL-10 and TGF- $\beta$ were determined by intracellular cytokine staining (12).

Murine autoimmune diabetes model. Autoimmune diabetes was induced in $\mathrm{F}_{1}$ mice that were crossed between RIP-mOVA Tg with OT-I TCR Tg mice by i.p. injection with VACV-OVA $\left(2 \times 10^{6} \mathrm{PFU} / \mathrm{mouse}\right)$ (49). All of the mice developed diabetes after viral injection. Blood glucose was measured 1 week after the VACV-OVA injection.

Blood glucose measurement. Blood glucose levels were determined using a Glucometer Ascensia Elite $\mathrm{XL}$ (Bayer). Six hundred milligrams per deciliter is the maximum measurable glucose reading. Mice were typically considered diabetic with readings of $>250 \mathrm{mg} / \mathrm{dl}$.

Adoptive cell transfer. B6-mOVA Tg $\times$ OT-I TCR double-Tg mice $\left(\mathrm{F}_{1}\right)$ were immunized with VACV-OVA for 1 week. At week 10, OVA-specific pre-iPSC-Tregs or iPSC-derived control cells $\left(3 \times 10^{6}\right)$ were i.v. trans- 
ferred into diabetic mice. Four to six weeks later, mice were euthanized, and the pancreatic tissues were removed for histopathological examination.

Histology and immunohistochemistry. For H\&E staining, pancreatic tissues were fixed with $10 \%$ neutral formalin solution (VWR), and the fixed samples were prepared and stained as described previously (48). For immunofluorescent microscopy, the pancreatic tissues were frozen in cryovials on dry ice immediately after resection. Cryosectioning and immunofluorescent staining were performed as described previously (48).

Statistics. Multiple Student's 1-tailed $t$ test or 1-way ANOVA or 2-way ANOVA analysis was performed to analyze the differences between the groups, using GraphPad Prism. A $P$ value of less than 0.05 was considered significant.

Study approval. The present studies in mice were reviewed and approved by The Texas A\&M University Animal Care Committee and were in accordance with the guidelines of the Association for the Assessment and Accreditation of Laboratory Animal Care.

\section{Author contributions}

JS and JMY designed the experiments, analyzed data, and contributed to the writing of the paper. $\mathrm{MH}$, FL, and JKD performed the experiments. DF and SSA provided reagents in the animal model. XX and $\mathrm{XR}$ analyzed data.

\section{Acknowledgments}

JS is the guarantor of this work and, as such, had full access to all the data in the study and takes responsibility for the integrity of the data and the accuracy of the data analysis. This work was supported by National Institutes of Health grants R01AI121180, R01CA221867, and R21AI109239 and the American Diabetes Association (1-16-IBS-281 to JS).

Address correspondence to: Jianxun Song, 1114 TAMU, 473A Reynolds Medical Building, College Station, Texas 77843-1114, USA. Phone:1.979.436.0633; Email: jus35@tamu.edu.

1. Delovitch TL, Singh B. The nonobese diabetic mouse as a model of autoimmune diabetes: immune dysregulation gets the NOD. Immunity. 1997;7(6):727-738.

2. Hori S, Nomura T, Sakaguchi S. Control of regulatory T cell development by the transcription factor Foxp3. Science. 2003;299(5609):1057-1061.

3. Hänninen A, Jalkanen S, Salmi M, Toikkanen S, Nikolakaros G, Simell O. Macrophages, T cell receptor usage, and endothelial cell activation in the pancreas at the onset of insulin-dependent diabetes mellitus. J Clin Invest. 1992;90(5):1901-1910.

4. Shull MM, et al. Targeted disruption of the mouse transforming growth factor-beta 1 gene results in multifocal inflammatory disease. Nature. 1992;359(6397):693-699.

5. Bottazzo GF, Dean BM, McNally JM, MacKay EH, Swift PG, Gamble DR. In situ characterization of autoimmune phenomena and expression of HLA molecules in the pancreas in diabetic insulitis. N Engl J Med. 1985;313(6):353-360.

6. Sakaguchi S, Miyara M, Costantino CM, Hafler DA. FOXP3+ regulatory T cells in the human immune system. Nat Rev Immunol. 2010;10(7):490-500.

7. Roncarolo MG, Battaglia M. Regulatory T-cell immunotherapy for tolerance to self antigens and alloantigens in humans. Nat Rev Immunol. 2007;7(8):585-598.

8. Tang $\mathrm{Q}$, et al. In vitro-expanded antigen-specific regulatory $\mathrm{T}$ cells suppress autoimmune diabetes. J Exp Med. 2004;199(11):1455-1465.

9. Wright GP, Ehrenstein MR, Stauss HJ. Regulatory T-cell adoptive immunotherapy: potential for treatment of autoimmunity. Expert Rev Clin Immunol. 2011;7(2):213-225.

10. Sekiya T, et al. Nr4a receptors are essential for thymic regulatory T cell development and immune homeostasis. Nat Immunol. 2013;14(3):230-237.

11. Haque R, et al. Programming of regulatory T cells from pluripotent stem cells and prevention of autoimmunity. J Immunol. 2012;189(3):1228-1236

12. Haque M, et al. Stem cell-derived tissue-associated regulatory T cells ameliorate the development of autoimmunity. Sci Rep. 2016;6:20588.

13. Belkaid Y, Piccirillo CA, Mendez S, Shevach EM, Sacks DL. CD4+CD25+ regulatory T cells control Leishmania major persistence and immunity. Nature. 2002;420(6915):502-507.

14. Lee I, Wang L, Wells AD, Dorf ME, Ozkaynak E, Hancock WW. Recruitment of Foxp3+ T regulatory cells mediating allograft tolerance depends on the CCR4 chemokine receptor. J Exp Med. 2005;201(7):1037-1044.

15. Kukreja A, et al. Multiple immuno-regulatory defects in type-1 diabetes. J Clin Invest. 2002;109(1):131-140.

16. Coppieters KT, et al. Demonstration of islet-autoreactive CD8 T cells in insulitic lesions from recent onset and long-term type 1 diabetes patients. J Exp Med. 2012;209(1):51-60.

17. Ihm SH, Yoon JW. Studies on autoimmunity for initiation of beta-cell destruction. VI. Macrophages essential for development of beta-cell-specific cytotoxic effectors and insulitis in NOD mice. Diabetes. 1990;39(10):1273-1278 
18. Lee KU, Amano K, Yoon JW. Evidence for initial involvement of macrophage in development of insulitis in NOD mice. Diabetes. 1988;37(7):989-991.

19. Calderon B, Carrero JA, Miller MJ, Unanue ER. Entry of diabetogenic T cells into islets induces changes that lead to amplification of the cellular response. Proc Natl Acad Sci USA. 2011;108(4):1567-1572.

20. Pober JS, et al. Activation of cultured human endothelial cells by recombinant lymphotoxin: comparison with tumor necrosis factor and interleukin 1 species. J Immunol. 1987;138(10):3319-3324.

21. Herman AE, Freeman GJ, Mathis D, Benoist C. CD4+CD25+ T regulatory cells dependent on ICOS promote regulation of effector cells in the prediabetic lesion. J Exp Med. 2004;199(11):1479-1489.

22. Chen Z, Herman AE, Matos M, Mathis D, Benoist C. Where CD4+CD25+ T reg cells impinge on autoimmune diabetes. $J$ Exp Med. 2005;202(10):1387-1397.

23. Campbell IL, Kay TW, Oxbrow L, Harrison LC. Essential role for interferon-gamma and interleukin-6 in autoimmune insulin-dependent diabetes in NOD/Wehi mice. J Clin Invest. 1991;87(2):739-742.

24. Sarvetnick N, Liggitt D, Pitts SL, Hansen SE, Stewart TA. Insulin-dependent diabetes mellitus induced in transgenic mice by ectopic expression of class II MHC and interferon-gamma. Cell. 1988;52(5):773-782.

25. Ben Nasr M, et al. PD-L1 genetic overexpression or pharmacological restoration in hematopoietic stem and progenitor cells reverses autoimmune diabetes. Sci Transl Med. 2017;9(416):eaam7543.

26. Ben Nasr M, et al. The use of hematopoietic stem cells in autoimmune diseases. Regen Med. 2016;11(4):395-405.

27. Ben Nasr M, Frumento D, Fiorina P. Adipose stem cell therapy for chronic pancreatitis. Mol Ther. 2017;25(11):2438-2439.

28. Fiorina P, et al. Targeting CD22 reprograms B-cells and reverses autoimmune diabetes. Diabetes. 2008;57(11):3013-3024.

29. Leiter EH, Schile A. Genetic and pharmacologic models for type 1 diabetes. Curr Protoc Mouse Biol. 2013;3(1):9-19.

30. Precopio ML, et al. Immunization with vaccinia virus induces polyfunctional and phenotypically distinctive CD8(+) $\mathrm{T}$ cell responses. JExp Med. 2007;204(6):1405-1416.

31. Di Genova G, Savelyeva N, Suchacki A, Thirdborough SM, Stevenson FK. Bystander stimulation of activated CD4+ T cells of unrelated specificity following a booster vaccination with tetanus toxoid. Eur J Immunol. 2010;40(4):976-985.

32. Whiteside SK, et al. IL-10 Deficiency reveals a role for TLR2-dependent bystander activation of T cells in lyme arthritis. $J$ Immunol. 2018;200(4):1457-1470.

33. Sakaguchi S. Naturally arising Foxp3-expressing CD25+CD4+ regulatory T cells in immunological tolerance to self and nonself. Nat Immunol. 2005;6(4):345-352.

34. Chatenoud L, Salomon B, Bluestone JA. Suppressor T cells--they're back and critical for regulation of autoimmunity! Immunol Rev. 2001;182:149-163.

35. Ehrenstein MR, et al. Compromised function of regulatory T cells in rheumatoid arthritis and reversal by anti-TNFalpha therapy. J Exp Med. 2004;200(3):277-285.

36. Huan J, et al. Decreased FOXP3 levels in multiple sclerosis patients. J Neurosci Res. 2005;81(1):45-52.

37. Morgan ME, et al. Effective treatment of collagen-induced arthritis by adoptive transfer of CD25+ regulatory T cells. Arthritis Rheum. 2005;52(7):2212-2221.

38. Kohm AP, Carpentier PA, Anger HA, Miller SD. Cutting edge: CD4+CD25+ regulatory T cells suppress antigen-specific autoreactive immune responses and central nervous system inflammation during active experimental autoimmune encephalomyelitis. J Immunol. 2002;169(9):4712-4716.

39. Scalapino KJ, Tang Q, Bluestone JA, Bonyhadi ML, Daikh DI. Suppression of disease in New Zealand Black/New Zealand White lupus-prone mice by adoptive transfer of ex vivo expanded regulatory T cells. J Immunol. 2006;177(3):1451-1459.

40. Tarbell KV, Yamazaki S, Olson K, Toy P, Steinman RM. CD25+ CD4+ T cells, expanded with dendritic cells presenting a single autoantigenic peptide, suppress autoimmune diabetes. J Exp Med. 2004;199(11):1467-1477.

41. Salomon B, et al. B7/CD28 costimulation is essential for the homeostasis of the CD4+CD25+ immunoregulatory T cells that control autoimmune diabetes. Immunity. 2000;12(4):431-440.

42. Bullard DC, Hu X, Crawford D, McDonald K, Ramos TN, Barnum SR. Expression of a single ICAM-1 isoform on T cells is sufficient for development of experimental autoimmune encephalomyelitis. Eur J Immunol. 2014;44(4):1194-1199.

43. Bullard DC, Hu X, Schoeb TR, Collins RG, Beaudet AL, Barnum SR. Intercellular adhesion molecule-1 expression is required on multiple cell types for the development of experimental autoimmune encephalomyelitis. J Immunol. 2007;178(2):851-857.

44. Korn T, et al. IL-21 initiates an alternative pathway to induce proinflammatory T(H)17 cells. Nature. 2007;448(7152):484-487.

45. Honkanen J, et al. IL-17 immunity in human type 1 diabetes. J Immunol. 2010;185(3):1959-1967.

46. Arif S, et al. Peripheral and islet interleukin-17 pathway activation characterizes human autoimmune diabetes and promotes cytokine-mediated $\beta$-cell death. Diabetes. 2011;60(8):2112-2119.

47. Ablamunits V, et al. Synergistic reversal of type 1 diabetes in NOD mice with anti-CD3 and interleukin-1 blockade: evidence of improved immune regulation. Diabetes. 2012;61(1):145-154.

48. Lei $\mathrm{F}$, et al. In vivo programming of tumor antigen-specific $\mathrm{T}$ lymphocytes from pluripotent stem cells to promote cancer immunosurveillance. Cancer Res. 2011;71(14):4742-4747.

49. Haque M, et al. C-Myc regulation by costimulatory signals modulates the generation of CD8+ memory T cells during viral infection. Open Biol. 2016;6(1):150208. 